\title{
Luxação coxofemoral traumática em cães e gatos: estudo retrospectivo (1997-2006)
}

\author{
Traumatic coxofemoral luxation in dogs and cats: retrospective study (1997-2006)
}

\author{
Anna Laeticia da Trindade Barbosa' João Eduardo Wallau SchosslerII
}

\section{RESUMO}

As luxações coxofemorais traumáticas são importantes afecções ortopédicas nos cães e gatos devido a grande frequência com que acometem esses animais. A principal causa são traumas ocasionados por atropelamentos, e a apresentação craniodorsal destaca-se dentre as demais. Podem ocorrer em qualquer raça, sexo e idade, porém os animais adultos são os mais frequentemente afetados. $O$ tratamento primário de escolha é a redução fechada. No entanto, em algumas circunstâncias, faz-se necessário o uso do método aberto/cirúrgico. O objetivo deste trabalho foi avaliar os casos de luxações coxofemorais traumáticas no Hospital Veterinário Universitário da Universidade Federal de Santa Maria. Para isso, foi realizado um estudo retrospectivo dos atendimentos cirúrgicos realizados entre 1997 e 2006 nesse hospital. Observou-se alta incidência das luxações traumáticas $(75,8 \%)$, sendo o traumatismo por automóveis a causa mais frequente $(58,6 \%)$, seguido pelas quedas $(17,3 \%)$, pelos agentes desconhecidos $(17,2 \%)$ e pelas brigas $(6,9 \%)$. A proporção canina/felina foi de 29:1, sendo os machos os mais acometidos em ambas as espécies: $65,5 \%$ dos cães e $100 \%$ dos gatos. Os animais mais predispostos eram da raça Poodle $(44,8 \%)$ e aqueles sem raça definida $(20,7 \%)$. Os tratamentos utilizados foram capsulorrafia (18,3\%), pino transarticular $(56,7 \%)$ e excisão artroplástica da cabeça e do colo femoral como método primário (25\%). A taxa de sucesso foi de $71,1 \%$ nas duas primeiras técnicas, mostrando-se eficientes na redução da luxação coxofemoral traumática, recomendando-se a excisão da cabeça e do colo femoral em sua impossibilidade ou em complicações.

Palavras-chave: articulação coxofemoral, deslocamento traumático, levantamento.

\section{ABSTRACT}

The traumatic coxofemoral luxations are important orthopedics injuries in dogs and cats due to their great frequency. The main causes are the automobilistic accidents and craniodorsal appearance is the most frequent. This injury can affect any breed, sex and age, however, adult animals are most frequently involved. Close reduction is the first choice of treatment, but at some circumstances the use of open/surgical methods are necessary. This study aims to evaluate the incidence, predisposition, etiology and results of treatments of traumatic hip luxation at the Santa Maria Federal University Veterinary Hospital. For this, a retrospective study of surgical service was made between 1997 and 2006 in this hospital. It was observed a high incidence of traumatic luxations (75.8\%), and the most frequent cause was hit $t$ by a car trauma (58.6\%), followed by falls (17.3\%), unknown agents (17.2\%) and fights (6.9\%). The canine/feline proportion was 58/2, with the males being the most affected in both species: $65.5 \%$ at dogs and $100 \%$ at cats. The most predicts breed involved was the Poodle (44.8\%) and the mixed breed dogs (20.7\%). The treatments options used were suture of capsule (18.3\%), transacetabular pinnig (56.7\%) and femoral head and neck excision (25\%). The success rate was $71.1 \%$ in the first two techniques, proving itself efficient in the traumatic coxofemoral luxation reduction, thus, femoral head and neck excision are recommended in complications and impossibility.

Key words: hip joint, traumatic dislocation, sense.

\section{INTRODUÇÃO}

As luxações são parte significativa dos casos ortopédicos observados na medicina veterinária em pequenos animais. A articulação coxofemoral é a mais frequentemente luxada no cão e no gato (WADSWORTH, 1996), sendo a freqüência no cão maior em animais com mais de 11 e 12 meses de idade (WALLACE, 1991). Não há ligamentos colaterais, e os

'Programa de Pós-graduação em Medicina Veterinária, Laboratório de Cirurgia Experimental (LACE), Universidade Federal de Santa Maria (UFSM), 97105-900, Santa Maria, RS, Brasil. E-mail: laetíciatrindade@yahoo.com.br. Autor para correspondência.

"Departamento de Clínica de Pequenos Animais (DCPA), Centro de Ciências Rurais (CCR), UFSM, Santa Maria, RS, Brasil. 
músculos que se fixam à extremidade proximal do fêmur permitem grande movimentação da articulação. $\mathrm{O}$ principal aspecto estabilizador dessa articulação é sua própria configuração esferoidal, onde o ligamento redondo e a cápsula articular são as principais estruturas de tecido mole que se contrapõem à luxação (WADSWORTH, 1996).

As luxações coxofemorais em cães e gatos geralmente resultam de traumas externos, sendo 59 a $83 \%$ devido a traumatismos produzidos por veículos. A maioria das lesões é unilateral e, devido às grandes forças requeridas para produzir a luxação, por volta de 50\% têm lesões importantes associadas, tais como traumatismos torácicos (BRINKER et al., 1999). São classificadas em dorsal, craniodorsal, caudodorsal, ventral, ventrocaudal, ventrocranial, ou ainda, intrapélvica, menos comum (WADSWORTH, 1996).

As luxações craniodorsais são as mais frequentes (DECAMP, 1995). Sua principal causa é um forte traumatismo aplicado à região glútea do animal, provocando sua queda na direção da articulação coxofemoral, sofrendo a luxação. Quando o trocânter maior colide com o solo, a energia do golpe é transmitida do colo femoral até a cabeça femoral. Esta é impulsionada por sobre a borda dorsal, lacerando a cápsula articular ou o ligamento redondo. Algumas vezes este sofre avulsão da cabeça femoral ou do acetábulo, podendo carregar algum fragmento ósseo consigo. Porém, raramente um fragmento ósseo é retirado da borda dorsal do acetábulo. Desse modo, a cabeça femoral vai repousar em sua posição mais comum de luxação, crânio-dorsalmente ao acetábulo (WADSWORTH, 1996).

A maioria dos animais tem histórico de trauma, geralmente atropelamento, e não suporta peso sobre o membro. No caso de luxação craniodorsal, a perna se aduz, sofrendo rotação externa (MANLEY, 1998). No exame físico, pode-se palpar uma distância maior do que a normal entre o trocânter maior do fêmur e a tuberosidade isquial (TOMLINSON, 1998). Ao se praticar uma extensão completa dos membros pélvicos, o membro afetado estará mais curto, em caso de luxação craniodorsal, e mais comprido nas ventrocaudais. A comparação com o lado não afetado frequentemente revela assimetria na posição dos trocânteres maiores (MANLEY, 1998). Uma crepitação pode ser sentida ao se colocar a mão sobre a articulação e com a outra rotar o membro (WHITTICK, 1978). No caso das ventrocaudais, o membro é comumente mantido em abdução com rotação interna. É essencial um exame radiográfico em dois posicionamentos, não apenas para o diagnóstico clínico, mas também para identificação de possíveis complicações, como fraturas da cabeça do fêmur, da borda dorsal do acetábulo, ou pélvicas (MANLEY, 1998).

Apesar das luxações coxofemorais não serem consideradas emergências, é preciso tratá-las o mais rápido possível (WALLACE, 1991). Caso contrário, formar-se-á uma pseudoartrose, resultando em graus variáveis de disfunção (MANLEY, 1998). O tratamento depende do deslocamento traumático ser recente ou antigo (WHITTICK, 1978). Quanto mais tempo o quadril estiver deslocado, mais difícil será sua redução, devido à contração muscular e à fibrose local (WADSWORTH, 1996).

A redução fechada deve ser o método de eleição em qualquer caso em que os danos aos tecidos moles, articulares ou ósseos sejam pequenos (WADSWORTH, 1996). Na maioria dos casos, desde que o acetábulo e a cabeça femoral não sejam displásicos, é satisfatória a redução fechada por tração direta (BARDET, 1997). Essa técnica pode ser utilizada até 48 horas após o trauma (WALLACE, 1991) ou dentro dos primeiros quatro ou cinco dias (BRINKER et al., 1999). Uma bandagem de Ehmer pode ser aplicada após as reduções fechadas craniodorsais, enquanto nas luxações caudoventrais as bandagens não são necessárias (MANLEY, 1998).

Se as técnicas de redução fechada falharem ou se o deslocamento reincidir com persistência depois de repetidas reduções, torna-se necessária uma redução aberta com fixação (BARDET, 1997). Devido à taxa de insucesso das reduções fechadas terem sido relatadas em torno de 47 a $65 \%$, alguns cirurgiões preferem partir direto para redução aberta e fixação (BRINKER et al., 1999).

A redução aberta é indicada em casos de avulsão da fóvea da cabeça femoral ou quando uma redução fechada não conseguiu manter a redução do quadril (JOHNSON \& HULSE, 2005). Se possível, a articulação coxofemoral deve ser reduzida antes do procedimento cirúrgico, o que facilita a dissecção. A escolha da abordagem varia com a situação. Se a articulação é redutível, a abordagem craniolateral é apropriada. Se necessário, pode ser expandida pela incisão do tendão do músculo glúteo (BRINKER et al., 1999).

A osteotomia do trocânter maior pela abordagem dorsal também pode ser usada (MANLEY, 1998). Essa abordagem é o método de escolha para luxações crônicas ou quando grande reconstrução é necessária. Depois que a articulação estiver exposta, os objetivos são remover ou reduzir qualquer tecido mole que se interponha ao acetábulo, reposicionar a cabeça femoral no acetábulo e estabilizá-la (BRINKER et al., 1999).

Ciência Rural, v.39, n.6, set, 2009. 
Várias opções estão disponíveis para manter a articulação estável. Se houver a cápsula articular intacta em cada lado da luxação, a simples sutura com fios absorvíveis sintéticos ou nãoabsorvíveis de maior calibre é suficiente (BRINKER et al., 1999). Se não houver a cápsula na qual as suturas possam ser ancoradas no lado acetabular ou no colo femoral, podem ser usados artefatos como parafusos ou outros materiais que possam ser fixados em osso e posteriormente no artefato fixado à sutura da cápsula articular. Quando a cápsula não puder ser fechada com segurança, medidas adicionais devem ser tomadas para assegurar a estabilidade da articulação, até que a cápsula seja reparada por fibroplastia (JOHNSON \& HULSE, 2005).

Estão disponíveis outros procedimentos cirúrgicos de estabilização da articulação coxofemoral, como a colocação de pino transarticular ou transacetabular (BRINKER et al., 1999), transposição trocantérica, sutura extracapsular, substituição do ligamento redondo (com inclusão de pinos), aplicação de enxerto ósseo na borda dorsal do acetábulo (WADSWORTH, 1996). Ainda há a técnica da cápsula sintética, fixação por pino em cavilha moldado (BRINKER et al., 1999), prótese plástica de nylon trançado ancorada atrás da fossa do acetábulo, por meio de uma cavilha (HICKMAN \& WALKER, 1983), estabilização por sutura absorvível extra-articular (MARTINI et al., 2001), substituição do ligamento da cabeça do fêmur com autoenxerto de fáscia lata (BRANDÃO et al., 2002), transposição do ligamento sacrotuberoso (KILIÇ et al., 2002), reconstituição do ligamento redondo com pericárdio bovino conservado em glicerina 98\% (RODASKI et al, 2002), reconstituição de cápsula articular com pericárdio bovino conservado em glicerina 98\% (BRANDÃO et al., 2006) e substituição do ligamento redondo por centro frênico equino conservado em glicerina 98\% (SIA et al., 2006).

A aplicação do pino transacetabular tem sido recomendada quando a cápsula articular está gravemente lesionada na luxação recidivante com a flexão e na extensão da articulação coxofemoral (MANLEY, 1998), em luxações recorrentes ou de longa duração. Para sua realização, localiza-se a fóvea da cabeça do fêmur e passa-se o pino através da fóvea em direção distal ao trocânter maior. Quando o pino apontar logo abaixo do trocânter maior, é puxado lentamente com auxílio do mesmo instrumento com que foi introduzido até que a ponta fique no nível da fóvea. $\mathrm{O}$ membro é então posicionado na sua posição anatômica, e o pino é introduzido com certa pressão em direção ao acetábulo, até emergir em torno de $6 \mathrm{~mm}$ dentro do canal pélvico. A ponta externa é cortada e dobrada sobre si.
Por toque retal pode ser confirmada a presença do pino dentro da cavidade pélvica (BRINKER et al., 1999).

O diâmetro do pino deve ser de aproximadamente dois terços ou três quartos da fóvea (TOMLINSON, 1998). Estudos realizados por ANDRIOLI (2000) mostraram que os pinos de diâmetro médio, correspondente a $17 \%$ da ocupação da cabeça femoral, são adequados para a manutenção da congruência articular sem ocasionar lesões visíveis à radiografia, que prejudiquem a deambulação normal.

A excisão da cabeça e do colo femoral para permitir a formação de pseudo-articulação fibrosa é também denominada excisão artroplástica ou ostectomia da cabeça e do colo femorais (BRINKER et al., 1999). Considerada um procedimento de salvamento, é recomendada nos casos de luxação recidivante realizada logo após métodos fechados e abertos de tratamento, presença de fraturas na cabeça e no colo femoral ou no acetábulo (MANLEY, 1998) e em luxações crônicas com alterações de cabeça femoral e acetábulo (FOX, 1991).

No pós-operatório das reduções abertas, pode-se instalar uma bandagem de Ehmer que sustente o peso (BRINKER et al., 1999), ou nenhuma bandagem adicional (WHITTICK, 1978). O exercício deve ser restrito durante as três primeiras semanas. No caso da fixação por pino transarticular, radiografias devem ser realizadas para assegurar a eficácia da técnica (MANLEY, 1998), e os pinos devem ser removidos com 14 a 21 dias de pós-operatório. As complicações dessa técnica são: quebra e migração do pino com penetração do cólon e sub ou re-luxação da articulação coxofemoral (BRINKER et al., 1999).

O prognóstico das reduções cirúrgicas varia com a estabilidade conseguida após a redução e com o intervalo de tempo entre a luxação e a redução. Casos que são reduzidos precocemente e com estabilidade adequada têm bom prognóstico, e a função essencialmente normal pode ser antecipada em 70 a 75\% desses animais (BRINKER et al., 1999).

O objetivo deste estudo foi avaliar a incidência, a predisposição, a etiologia e os tratamentos das luxações coxofemorais traumáticas em cães e gatos atendidos no Hospital Veterinário Universitário da Universidade Federal de Santa Maria.

\section{MATERIAL E MÉTODOS}

Foi realizado um estudo retrospectivo dos animais que apresentavam luxação coxofemoral traumática com indicação cirúrgica no período de janeiro de 1997 a janeiro de 2006, a partir do arquivo de pacientes encaminhados ao setor de cirurgia do 
Hospital Veterinário Universitário da Universidade Federal de Santa Maria. Os dados foram analisados considerando-se a espécie, a raça, o sexo, a idade, a etiologia do trauma, o tipo de luxação adquirida pelo deslocamento da cabeça femoral em relação ao acetábulo, os traumas concomitantes, as recidivas e suas complicações associadas. O tratamento utilizado também foi estudado e classificado de acordo com as diferentes condutas clínico-cirúrgicas. A claudicação pós-operatória não pôde ser avaliada por não ser um dado disponível na ficha de todos os animais

\section{RESULTADOS E DISCUSSÃO}

Durante 10 anos de estudo retrospectivo, foram atendidos 128 casos de luxação coxofemoral, em que $97(75,8 \%)$ eram compostos por luxações coxofemorais traumáticas, confirmando o traumatismo como uma das causas mais comuns de luxações coxofemorais (WADSWORTH, 1996). Destes, 24 eram constituídos de luxações associadas à fratura de cabeça e colo femorais e 13 associados à fratura acetabular, reforçando a importância do exame radiográfico para descartar lesões que apresentam os mesmos sinais clínicos, mas que não responderam ao tratamento para luxação (BRINKER et al., 1999). Assim, este estudo baseou-se nos 60 casos restantes, em que ocorreu somente a luxação coxofemoral traumática.

A predisposição por espécie e sexo demonstrou a ocorrência de 58 caninos e dois felinos. Desse total, 38 caninos (65,5\%) e dois felinos (100\%) eram machos e 20 caninos eram fêmeas. Porém, com relação aos felinos, nada pôde ser concluído devido ao pequeno número de animais. Esse grande número de animais da espécie canina também foi relatado por BASHER et al. (1986), ANDRIOLI (2000) e FREUND (2007), sem uma explicação específica. Uma das possíveis explicações para essa maior ocorrência de machos seria o fato desses animais saírem mais para as ruas, principalmente em busca de fêmeas no cio, portanto mais predispostos a serem atropelados ou se envolverem em brigas com outros machos pela fêmea, as duas maiores causas de luxação coxofemoral traumática.

Com relação à faixa etária dos cães, observou-se incidência entre três meses e 14 anos, estando $15,5 \%$ dos animais na faixa menor que um ano de idade, 53,5\% entre um e cinco anos, $24,1 \%$ entre cinco e 10 anos e 6,9\% acima de 10 anos. Esses dados confirmam que a frequência no cão é maior naqueles animais com mais de 11 e 12 meses de idade (WALLACE, 1991). A baixa incidência de luxações em animais jovens reflete o fato da fusão entre a cabeça e o colo femoral ocorrer entre 11 e 12 meses nos cães, portanto um trauma nesse período provocaria mais facilmente fratura epifisária (BASHER et al., 1986; FOX, 1991). A incidência reduzida nos cães acima de 10 anos pode ser devido aos animais mais idosos terem uma vida mais sedentária, portanto menos predispostos a traumas. Outro fato que poderia justificar esse baixo índice seria que, na senilidade, os ossos tendem a se descalcificar e enfraquecer, o que, em caso de trauma, levaria a fraturas ao invés de luxações.

As luxações coxofemorais traumáticas podem afetar qualquer raça, idade e sexo (JOHNSON \& HULSE, 2005); porém, neste estudo, verificou-se predisposição de algumas raças, observação também descrita por ANDRIOLI (2000). Os cães acometidos apresentaram a seguinte distribuição racial: Poodle (44,8\%), sem raça definida (20,7\%), Boxer (5,2\%), Border Collie, Cocker Spaniel, Fox Terrier, Pastor Alemão, Pastor Belga e Pinscher (3,45\%), Dog Alemão, Labrador Retriever, Pointer e Rotweiler (1,72\%).

Dentre os gatos, nada pode ser concluído a respeito da incidência devido ao número muito pequeno, apenas dois animais. A causa dos Poodles terem sido os mais acometidos pode estar relacionada ao porte de anormalidades anatômicas ou à instabilidade articular muitas vezes encontrada em animais de raças “toy” (BONE et al., 1984); porém, também pode estar relacionada à grande quantidade de cães da raça Poodle existentes no Brasil. O fato de os animais sem raça definida serem o segundo grupo mais acometido demonstra que a enfermidade não é característica de uma raça especial (THACHER \& SCHRADER, 1985).

A respeito da etiologia do trauma nos cães, $58,6 \%$ foram atribuídos aos atropelamentos, concordando com os dados da literatura que reportam os acidentes automobilísticos como a causa mais comum de luxações, responsáveis por 50 a 80\% dos atendimentos (FOX, 1991; MEIJ et al., 1992). Os demais agentes etiológicos foram classificados como quedas (17,3\%), causas desconhecidas (17,2\%) e brigas (6,9\%). Nos gatos, $100 \%$ da etiologia tinham caráter desconhecido. As causas desconhecidas, segundo BONE et al. (1984), podem ser atribuídas a acidentes automobilísticos, pois muitas vezes os proprietários relatam que o animal saiu de casa e voltou claudicando.

Quanto ao tipo de luxação adquirida pelo deslocamento da cabeça femoral em relação ao acetábulo, as mais frequentes foram as craniodorsais, somando $90 \%$ dos casos, seguidas pelas caudodorsais, com $6,7 \%$, e as caudoventrais, em 3,3\% dos pacientes. Fato condizente com citações anteriores que elegem a luxação craniodorsal como o deslocamento que ocorre 
na maioria das luxações (DECAMP, 1995). Em 91,7\% dos animais ocorreu luxação unilateral, dados semelhantes aos encontrados por EVERS et al. (1997) e FREUND (2006), os quais observaram luxação unilateral em 95,3\% e 97,2\% dos casos, respectivamente.

Devido às grandes forças requeridas para produzir a luxação, por volta de $50 \%$ têm lesões importantes associadas, principalmente traumatismo torácico (BRINKER et al., 1999). Porém, neste estudo, ocorreram traumas concomitantes somente em $20 \%$ dos casos, sendo os de maior frequência as fraturas de fêmur $(33,4 \%)$, seguidas das fraturas de pelve e luxações de patela (25\%), fraturas de tíbia e fíbula e pneumotórax (8,3\%).

Nos métodos de tratamento das luxações coxofemorais traumáticas, constam as reduções fechadas e abertas, sendo as reduções fechadas a terapia de escolha inicial. Situações em que a articulação permanece muito instável após redução ou a cabeça femoral não pode ser reduzida requerem abordagem aberta. Devido à taxa de insucesso das reduções fechadas terem sido relatadas em torno de 47 a $65 \%$, alguns cirurgiões preferem partir direto para redução aberta e fixação (BRINKER et al., 1999). Por este motivo este trabalho abordou somente os casos com indicação cirúrgica.

Os tratamentos cirúrgicos de redução da luxação coxofemoral usados foram escolhidos de acordo com a situação clínico-cirúrgica apresentada em cada caso e a preferência do cirurgião. Dessa forma, os métodos utilizados foram capsulorrafia (18,3\%), fixação por pino transarticular $(56,7 \%)$ e excisão artroplástica da cabeça e do colo femoral (25\%). Dos 60 pacientes operados, 45 animais (75\%) passaram por capsulorrafia ou fixação por pino transarticular. Destes, 13 animais sofreram dois procedimentos cirúrgicos devido à recidiva de um dos procedimentos citados anteriormente, sendo submetidos posteriormente à excisão artroplástica da cabeça e do colo femoral (Tabelas 1 e 2).
A capsulorrafia é um método eficiente, pois, devido à composição firme e espessa da cápsula articular, é uma das estruturas que confere estabilidade à articulação coxofemoral, porém a técnica só pode ser utilizada como método primário quando a cápsula não estiver muito lesionada (MANLEY, 1998). Acredita-se que o baixo percentual de uso dessa técnica verificado neste estudo está relacionado com a ruptura parcial ou total da cápsula nos demais casos, impossibilitando sua reconstituição. Os resultados obtidos com essa técnica neste estudo foram satisfatórios, confirmando a eficiência da técnica, já que foi efetiva em 72,7\% dos casos em que foi utilizada.

A aplicação do pino transarticular tem sido recomendada quando a cápsula articular está gravemente lesionada ou quando ocorre luxação recidivante com a flexão e extensão da articulação (MANLEY, 1998). A explicação para ter sido a técnica mais utilizada está relacionada ao fato de ser a próxima indicação (das técnicas utilizadas neste estudo), quando a capsulorrafia não puder ser realizada, portanto, mais indicada que a excisão artroplástica.

A excisão artroplástica da cabeça e do colo femoral se restringe aos casos de luxação crônica com alterações de cabeça femoral e acetábulo (FOX, 1991), também considerada um procedimento de salvamento, quando todas as outras técnicas falharam (MANLEY, 1998). O uso dessa técnica compreendeu 15 casos em que foi observada desvitalização da cabeça femoral, três pacientes que apresentaram re-luxação após uso da capsulorrafia e 10 pacientes com re-luxação após fixação por pino transarticular.

Em todos os casos avaliados neste estudo, não foi instalada nenhuma bandagem adicional conforme WHITTICK, (1978), aparentemente sem comprometimento do resultado final. Porém, discuti-se se os casos de recidiva após sutura capsular poderiam ter sido minimizados com o uso da bandagem.

Dos 45 animais tratados pelas técnicas de capsulorrafia e pino transarticular, 13 animais (28,9\%) apresentaram re-luxação, confirmação de que os métodos abertos possuem percentuais de sucesso em torno de 70 a 100\% (ALVARENGA et al., 1997).

Tabela 1 - Demonstrando o número de animais e a porcentagem de cada procedimento cirúrgico desenvolvido a partir do número de pacientes submetidos à cirurgia da articulação coxofemoral em um primeiro momento.

\begin{tabular}{ccl}
\hline Número de Pacientes & Porcentagem (\%) & \multicolumn{1}{c}{ Procedimentos Cirúrgicos } \\
\hline 60 & 100 & $\begin{array}{l}\text { Capsulorrafia } \\
\text { Inserção de Pino Transacetabular } \\
\text { Excisão Artroplástica da cabeça e do colo femoral (como método primário) } \\
\text { Capsulorrafia }\end{array}$ \\
11 & 18,3 & Inserção de Pino Transacetabular \\
34 & 56,7 & Excisão Artroplástica da cabeça e do colo femoral (como método primário) \\
\hline
\end{tabular}

Ciência Rural, v.39, n.6, set, 2009. 
Tabela 2 - Demonstrando o número e a porcentagem de pacientes que sofreram recidiva após as cirurgias de Capsulorrafia e Inserção de Pino Transacetabular, sendo submetidos posteriormente à Excisão Artroplástica da cabeça e do colo femoral em um segundo momento.

\begin{tabular}{ccl}
\hline Número de Pacientes & Porcentagem (\%) & \multicolumn{1}{c}{ Procedimentos Cirúrgicos } \\
\hline 45 & 100 & $\begin{array}{l}\text { Capsulorrafia } \\
\text { Inserção de Pino Transacetabular } \\
\text { Excisão Artroplástica da cabeça e do colo femoral (como método } \\
\text { secundário após recidiva) }\end{array}$ \\
\hline
\end{tabular}

As recidivas aconteceram em $27,3 \%$ dos pacientes após a capsulorrafia e em $29,4 \%$ após a inserção de pino transarticular. Somente em $23,0 \%$ dos casos recidivantes (três animais) foram observadas complicações associadas à instabilidade como desenvolvimento de necrose asséptica da cabeça femoral (7,7\%), infecção (7,7\%) e fratura da cabeça e do colo femorais $(7,7 \%)$. Não foram observadas a quebra e migração do pino com penetração do cólon como descritos na literatura (BRINKER et al., 1999). Os demais casos recidivantes não foram observadas complicações associadas à re-luxação.

\section{CONCLUSÕES}

As luxações coxofemorais traumáticas possuem grande importância dentre as afecções ortopédicas em pequenos animais devido a sua alta incidência. Cães machos e adultos da raça Poodle foram os pacientes mais acometidos, sendo os atropelamentos a etiologia de maior freqüência. A capsulorrafia e fixação por pino transarticular são técnicas eficientes na redução da luxação coxofemoral traumática. No entanto, diante de sua impossibilidade ou complicações recomenda-se a excisão artroplástica da cabeça e do colo femoral.

\section{REFERÊNCIAS}

ALVARENGA, J. et al. Luxação coxofemoral recidivante em cães. Revista Brasileira de Ciências Veterinárias, v.4, n.2, p.45-53, 1997.

ANDRIOLI, L.G. Luxações coxofemorais traumáticas. Análise da técnica de fixação transarticular e dos aspectos relacionados ao diâmetro ideal do pino. 2000. 38f. Dissertação (Mestrado em Cirurgia) - Curso de Pós-graduação em Medicina Veterinária, Universidade Federal de Santa Maria.

BARDET, J.F. Claudicação. In: ETTINGER, S.J.; FELDMAN, E.C. Tratado de medicina interna veterinária. Moléstias do cão e do gato. 4.ed. São Paulo: Manole, 1997. Cap.33, p.188-196.

BASHER, A.W.P. et al. Coxofemoral luxation in the dog and cat. Veterinary Surgery, v.15, n.5, p.356-362, 1986.
BONE, D.L. et al. Traumatic coxofemoral luxation in dogs: Results of repair. Veterinary Surgery, v.13, p.263-270, 1984.

BRANDÃO, C.V.S. et al. Substituição do ligamento da cabeça do fêmur com auto-enxerto de fáscia lata na luxação coxofemoral em cães. Ciência Rural, v.32, n.2, p.275-280, 2002.

BRANDÃO, C.V.S. et al. Avaliação macro e microscópica da reconstituição da cápsula articular utilizando pericárdio bovino na luxação coxofemoral experimental em cães. Veterinária e Zootecnia, v.13, n.1, p.73-83, 2006.

BRINKER, W.O. et al. Tratamento das luxações coxofemorais. In:_____. Manual de ortopedia e tratamento das fraturas dos pequenos animais. São Paulo: Manole, 1999. Cap.15, p.394-406.

DECAMP, C.E. Dislocations. In: OLMSTEAD, M. Small animal orthopedics. St. Louis: Mosby, 1995. Cap.16, p.347353.

EVERS, P. et al. Long terms results of treatment of traumatic coxofemoral joint dislocation in dogs: 64 cases. Journal American Veterinary Medical Association, v.210, p.5964, 1997.

FOX, E.M. Coxofemoral luxations in dogs. Compendium European Edition, v.13, p.153-162, 1991.

FREUND, J.T. Luxatio ossis femoris traumatica bei hund und katze. Capturado em 8 maio 2007. Online. Disponível na Internet http://library.vetmed.fu-berlin.de/diss-abstract/ 116044.html.

HICKMAN, J.; WALKER, R.G. Atlas de cirurgia veterinária. 2.ed. Rio de Janeiro: Guanabara Koogan, 1983. 236p.

JOHNSON, A.L.; HULSE, D.A. Artropatias. In: FOSSUM, T.W. Cirurgia de pequenos animais. 2.ed. São Paulo: Roca, 2005. Cap.35, p.1095-1101.

KILIÇ, E. et al. Transposition of the sacrotuberous ligament for the treatment of coxofemoral luxation in dogs. Journal of Small Animal Practice, v.43, p.341-344, 2002.

MANLEY, P.A. Articulação coxofemoral. In: SLATTER, D. Manual de cirurgia de pequenos animais. 2.ed. São Paulo: Manole, 1998. Cap.135, p.2113-2133.

MARTINI, F.M et al. Extra-articular absorbable suture stabilization of coxofemoral luxation in dogs. Veterinary Surgery, v.30, p.468-475, 2001. 
MEIJ, B.P. et al. Results of extra-articular stabilization following open reduction of coxofemoral luxation in dogs and cats. Journal of Small Animal Practice, v.33, p.320-326, 1992.

RODASKI, S. et al. Artroplastia acetábulo-femoral em cães com pericárdio bovino conservado. Archives of Veterinary Science, v.7, n.2, p.179-187, 2002.

SIA, D.B. et al. Substituição do ligamento redondo por membrana biológica e pino transarticular na redução de luxação coxofemoral bilateral em cães. Acta Scientiae Veterinariae, v.34, n.2, p.187-192, 2006.

THACHER, C.; SCHRADER, S. Caudal ventral hip luxation in the dog: a review of 15 cases. Journal of the American Animal Hospital Association, v.21, p.167-172, 1985.
TOMLINSON JR, J.L. Reduction of coxofemoral luxations. In: BOJRAB, M.J. Current techniques in small animal surgery. Baltimore: Williams \& Wilkins, 1998. p.11781185.

WADSWORTH, P.L. Biomecânica das luxações. In: BOJRAB, M.J. Mecanismo da moléstia na cirurgia dos pequenos animais. São Paulo: Manole, 1996. Cap.139, p.1213-1216.

WALLACE, L.J. Técnicas de colocação de pinos para o reparo das luxações coxofemorais. In: BOJRAB, M.J. Cirurgia dos pequenos animais. São Paulo: Roca, 1991. p.660-666.

WHITTICK, W.G. Traumatologia y ortopedia canina. Barcelona: Aedos, 1978. 418p. 